\title{
Peak Work Rate during Exercise Could Detect Frailty Status in Elderly Patients with Stable Heart Failure
}

\author{
Kazuhiro Kawashima, ${ }^{1}$ MD, Akihiro Hirashiki ${ }^{1,2}$ MD, Kenichiro Nomoto, ${ }^{1}$ MD, Manabu Kokubo, ${ }^{1}$ MD, \\ Atsuya Shimizu, ${ }^{1}$ MD, Takashi Sakurai, ${ }^{1}$ MD, Izumi Kondo, ${ }^{1}$ MD, Yukihiko Washimi, ${ }^{1}$ MD, \\ Hidenori Arai, ${ }^{1} \mathrm{MD}$, Kenji Toba, ${ }^{1} \mathrm{MD}$ and Toyoaki Murohara, ${ }^{2} \mathrm{MD}$
}

\begin{abstract}
Summary
The Kihon Checklist (KCL) is a reliable tool for determining frailty status in the elderly. However, there is no information in the literature about the relationship between frailty status and exercise capacity. Here, we examined the associations between cardiopulmonary exercise testing parameters and frailty status in elderly patients with stable heart failure (HF).

Ninety-two elderly patients with stable HF were evaluated using cardiopulmonary exercise testing and the KCL. A KCL score of 0-3 was classified as robust, 4-7 as pre-frail, and $\geq 8$ as frail.

Mean age, peak $\mathrm{VO}_{2}$, and KCL score were 81.7 years, $13.2 \mathrm{~mL} / \mathrm{kg} /$ minute, and 10.7 , respectively. KCL score was significantly correlated with peak $\mathrm{VO}_{2}(r=-0.527, P<0.001)$ and peak work rate $(r=-0.632, P<$ 0.001). In patients with frailty $(n=63)$, the peak work rate (WR) was significantly lower than it was in patients without frailty $(n=29 ; 39.9$ versus $69.5 \mathrm{~W}$, respectively; $P<0.001)$. Multivariate analysis revealed that peak WR and peak systolic blood pressure were significant, independent predictors of frailty $(\beta=-0.108$ and -0.045 , respectively). In a diagnostic performance plot analysis, a cutoff value for peak WR of $51.9 \mathrm{~W}$ was the best predictor of frailty.

Frailty status was significantly associated with peak WR and peak systolic blood pressure in elderly patients with stable HF. Therefore, cardiopulmonary exercise testing may be useful for assessing frailty status in this patient population.
\end{abstract}

(Int Heart J 2019; 60: 1366-1372)

Key words: Cardiopulmonary exercise testing, Peak systolic blood pressure

$\mathrm{F}$ railty is a syndrome associated with aging that produces subclinical dysfunction across multiple organ systems, leading to increased risk of mortality. ${ }^{1)}$ It has been reported that $25-50 \%$ of patients with cardiovascular disease are frail. ${ }^{2)}$ The Kihon Checklist (KCL) was developed by the Japanese Ministry of Health, Labour and Welfare to identify older persons with frailty in need of care; it is a reliable tool for predicting general frailty in the elderly. ${ }^{3)}$

Cardiopulmonary exercise testing (CPX) is an established assessment tool in heart failure (HF) populations. ${ }^{4}$ A major advantage of CPX is that it provides an accurate measurement of exercise capacity; the degree to which ventilation is abnormally heightened during exercise is directly related to HF severity and a strong marker of prognosis. ${ }^{4)}$ In addition, CPX can be performed with adjunctive imaging modalities for diagnostic assessment, ${ }^{5,6)}$ and it has already proven useful for diagnosing $\mathrm{HF}^{4)}$

Given the emerging importance of detecting frailty and sarcopenia in an increasing elderly HF population, 2,7) and the already established role of CPX in the HF setting, determining the suitability of using CPX to identify patients with frailty is an important research endeavor. However, to date, there are no studies in the literature examining the associations between CPX parameters and frailty in HF patients.

Here, we evaluated using CPX parameters to detect frailty in elderly patients with stable HF.

\section{Methods}

Study population: Ninety-two patients over age 65 , who were hospitalized for worsening HF at the Department of Cardiology at the National Center for Geriatrics and Gerontology, Obu, Japan, between August 2016 and July 2018, were enrolled in the study. Worsening HF was defined as pulmonary venous congestion or edema on chest $\mathrm{X}$-ray plus any symptoms (e.g. breathlessness, ankle swel-

From the ${ }^{1}$ Department of Cardiology, National Center for Geriatrics and Gerontology, Obu, Japan and ${ }^{2}$ Department of Cardiology, Nagoya University Graduate School of Medicine, Nagoya, Japan.

This study was supported by the 2016-2018 Ministry of Health, Labour and Welfare geriatrics and gerontology sponsored-research funds.

Address for correspondence: Akihiro Hirashiki, MD, Department of Cardiology, National Center for Geriatrics and Gerontology, 7-430, Morioka-cho, Obu, Aichi 474-8511, Japan. E-mail: hirasiki@ncgg.go.jp

Received for publication March 6, 2019. Revised and accepted May 29, 2019.

Released in advance online on J-STAGE November 15, 2019.

doi: 10.1536/ihj.19-120

All rights reserved by the International Heart Journal Association. 


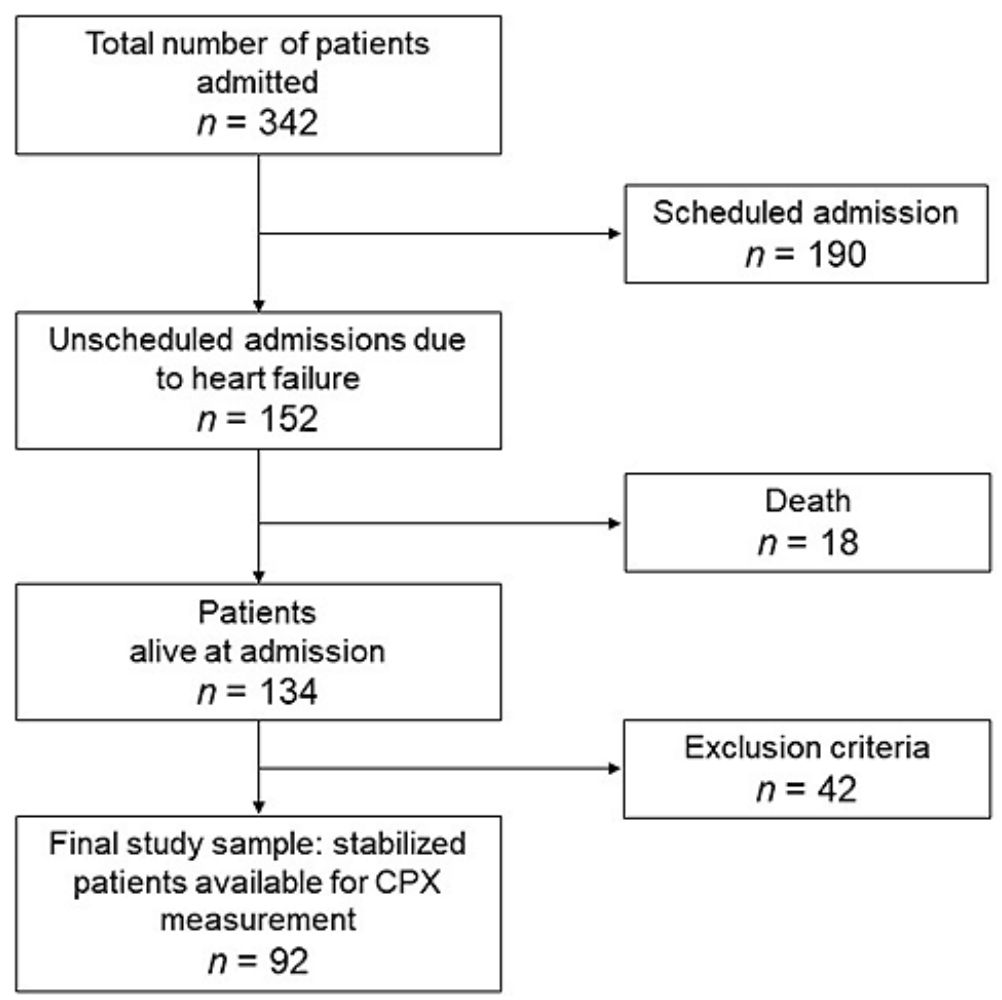

Figure 1. Study flow for the present analysis. Of the 342 patients admitted to the cardiology department of our hospital, 92 were included in the present study. CPX indicates cardiopulmonary exercise testing.

ling and fatigue) that may be accompanied by signs (e.g. elevated jugular venous pressure, pulmonary crackles, and peripheral edema).

Exclusion criteria were severe dementia, pulmonary dysfunction, hepatic dysfunction, end-stage renal dysfunction, malignant tumor prognosis within one year after hospitalization or discharge, inability to walk $10 \mathrm{~m}$, with or without using auxiliary equipment at discharge, and MiniMental State Exam fewer than 18 points.

Only patients who were stable after admission were enrolled (Figure 1). Of 152 patients with unscheduled hospital admittance due to worsening HF, 92 patients were included in the final analysis.

Study protocol: Physical examination, laboratory measurements, CPX, and the KCL questionnaire were performed within three days of study enrollment. All patients were in stable condition at the time of testing. The study protocol complied with the Declaration of Helsinki, and written informed consent was obtained from each subject. The ethics review board of the National Center for Geriatric and Gerontology approved the study (approval no. 1090).

KCL: The KCL is a 25 -item, self-administered questionnaire developed by the Japanese Ministry of Health, Labour and Welfare to identify elderly individuals who are at risk of requiring new certification for long-term care insurance. ${ }^{8)}$ The KCL comprises seven categories of questions that assess instrumental activities of daily living, physical function, nutritional status, oral function, social activities, cognitive function, and depressive mood. Thus, the KCL is a comprehensive evaluation tool that examines the social, psychological, and physical aspects of frailty. Given its ability to assess frailty across multiple domains, it regarded as an effective screening tool. ${ }^{9}$ The KCL questions require a simple yes or no answer, and are scored as 1 or 0 points, respectively. A KCL score of $0-3$ is classified as robust, $4-7$ as pre-frail, and $\geq 8$ as frail. ${ }^{9}$

CPX procedure: Each patient underwent CPX, at a progressively increasing work rate, to maximal tolerance on a cycle ergometer. The test protocol was conducted in accordance with the recommendations of the American Thoracic Society and American College of Chest Physicians. ${ }^{10)}$ The oxygen and carbon dioxide sensors were calibrated before each test with known oxygen, nitrogen, and carbon dioxide concentrations. The flow sensor was also calibrated before each test with a clean, empty 3-L syringe. Test termination criteria were patient request, volitional fatigue, ventricular tachycardia, $\geq 2 \mathrm{~mm}$ horizontal or downsloping ST-segment depression, or a drop in systolic blood pressure (SBP) of $\geq 20 \mathrm{mmHg}$ during exercise. A qualified exercise physiologist conducted each test under a physician's supervision. A 12-lead electrocardiogram was monitored continuously, and blood pressure was measured every minute during exercise and throughout the fiveminute recovery period. Respiratory gas exchange variables, including $\mathrm{VO}_{2}, \mathrm{VCO}_{2}$, and minute ventilation (VE), were acquired continuously throughout the test with an Oxycon Pro ergospirometer (Care Fusion; San Diego, CA, 
USA); gas-exchange data were obtained breath-by-breath. Peak $\mathrm{VO}_{2}$ and the peak respiratory exchange ratio were determined as the highest 30 -second average values obtained during the final stage of the test. The ratio of the increase in $\mathrm{VO}_{2}$ to the increase in work rate (WR) $\left[\Delta \mathrm{O}_{2} /\right.$ $\Delta \mathrm{WR}]$ was calculated by least-squares linear regression from the data recorded between 30 seconds after the start of the incremental exercise and 30 seconds before the end of the exercise.

Statistical analysis: Data are presented as means \pm standard deviation, unless otherwise stated. Variables were compared between HF-with-frailty and HF-without-frailty groups by using the Student's $t$-test for unpaired data. The chi-squared test was used to assess the significance of differences between dichotomous variables. Pearson's correlation coefficient was used to assess relationships between the KCL scores and CPX variables. We used univariate and multivariate linear regression analyses to identify factors that were independently associated with KCL score; the multivariate model included all baseline variables that had a significant correlation with KCL score in the univariate analysis. A diagnostic performance plot analysis was conducted to assess the utility of peak WR to distinguish between the presence (KCL score $\geq 8$ ) or absence of frailty. Cumulative frequency distributions were generated to determine the optimal cutoff point for detecting frailty. Sensitivity, specificity, accuracy, and positive predictive value were calculated using standard formulas. All analyses were performed with the SPSS 17.0 software package (SPSS; Chicago, IL, USA). A $P$ value of $<0.05$ was considered statistically significant.

\section{Results}

Patient characteristics: The patients' baseline clinical characteristics and a comparison between the non-frail and frail patients are shown in Table I. This study enrolled 92 consecutive elderly patients with HF (54 men (59\%); mean age $\pm \mathrm{SD}, 81.7 \pm 6.6$ years $)$. At the time of enrollment, all patients were stable and on optimal pharmacological therapy according to current HF treatment guidelines. ${ }^{11)}$ The mean (25th, 75th percentile) plasma brain natriuretic peptide (BNP) level was 182 (42, 272) $\mathrm{pg} / \mathrm{mL}$, and the mean left ventricular ejection fraction was $57.8 \% \pm 14.3 \%$. Based on the KCL, $68.4 \%$ of the patients had frailty (mean KCL score for all patients, $10.7 \pm 5.7$ ).

Subjects were allocated to one of two groups based on the absence $(n=29)$ or presence $(n=63)$ of frailty. Although there were no significant differences in underlying disease and medication use between the two groups, age was significantly higher in the frail group than in the non-frail group $(P=0.019)$. Similarly, plasma BNP level was significantly higher in the frail group than in the nonfrail group $(P=0.038)$; however, estimated glomerular filtration rate was comparable in both groups.

Peak $\mathrm{VO}_{2}$, peak $\mathrm{VO}_{2} / \mathrm{HR}$ ratio, peak WR, and $\Delta \mathrm{VO}_{2} /$ $\triangle \mathrm{WR}$ were significantly lower in the frail group than in the non-frail group $(P=0.006,0.002,<0.001,<0.001$, respectively). $\mathrm{VE} / \mathrm{VCO}_{2}$ slope was significantly higher in the frail group than in the non-frail group $(P=0.003)$. Although resting heart rate and resting SBP were compara- ble in both groups, peak HR and peak SBP during exercise were significantly lower in the frail group than in the non-frail group ( $P=0.021$ and 0.044 , respectively).

CPX parameters for detecting frailty: All CPX parameters during exercise, both at anabolic threshold and peak, were significantly correlated with KCL score (Table II). Of these variables, peak WR, peak $\mathrm{VO}_{2}, \mathrm{VE} / \mathrm{VCO}_{2}$ slope, $\Delta \mathrm{VO}_{2} / \Delta \mathrm{WR}$, and peak SBP were included in a multivariate analysis to determine their association with KCL score. Ultimately, peak WR and peak SBP were found to be significant independent predictors of frailty $(\beta$ : -0.108 , 95\% CI: -0.144 to $-0.072, P<0.001 ; \beta$ : $-0.045,95 \%$ CI, -0.074 to $-0.016, P=0.003$, respectively) (Table III).

Finally, we performed a diagnostic performance plot analysis to determine the peak WR cutoff value for predicting frailty (KCL score $\geq 8$; Figure 2 ); a peak WR cutoff value of $51.9 \mathrm{~W}$ was found to be the best predictor of frailty (accuracy, 0.706; positive predictive value, 0.821; negative predictive value, 0.557 ).

\section{Discussion}

Here, we report, for the first time, that reduced peak WR and peak SBP were strongly associated with KCL frailty status in elderly patients with $\mathrm{HF}$. Other $\mathrm{CPX}$ variables, including peak $\mathrm{VO}_{2}, \mathrm{VE} / \mathrm{VCO}_{2}$ slope, and $\Delta \mathrm{VO}_{2} /$ $\Delta \mathrm{WR}$, were also associated with frailty status. These results indicate that CPX variables, especially peak WR and peak systolic BP, could be important for diagnosing frailty in elderly patients with HF.

Several measurement tools have been developed to assess and measure frailty; however, there remains no internationally agreed upon standard tool. The KCL consists of simple questions and does not involve any special examinations, such as muscle strength or CPX. KCL scores have been shown to correlate significantly with Fried's frailty phenotype values. ${ }^{12)}$ Satake, et al. compared the diagnosis of frailty severity, based on the KCL criteria and the Cardiovascular Health Study (CHS) criteria, and reported sensitivities and specificities of $70.3 \%$ and $78.3 \%$ for pre-frail individuals and $89.5 \%$ and $80.7 \%$ for frail individuals, respectively. ${ }^{9)}$ In the present study, the KCL score and the Japanese version of the CHS (J-CHS) had a concordance rate of $77 \%$. We chose the KCL score to assess frailty severity. However, since associations between the KCL score and physical function in HF patients have not been examined, it remains unclear whether the KCL is also useful for assessing elderly patients with HF. Therefore, in the present study, we focused on evaluating CPX parameters' clinical usefulness for detecting frailty in elderly patients with stable HF.

Exercise testing remains a remarkably versatile tool that provides valuable diagnostic and prognostic information of patients with cardiovascular or pulmonary disease. ${ }^{4)}$ Although there are several assessment tools for detecting exercise capacity, CPX is one of the most valuable because taxing the mechanisms responsible for external and internal respiration by exercise can frequently reveal abnormalities not apparent at rest. ${ }^{4)}$ Although the mechanisms underlying the development of frailty in the elderly $\mathrm{HF}$ population remain unclear, it is easy to imagine over- 
Table I. Baseline Characteristics and Comparison between Non-Frail and Frail Patients

\begin{tabular}{|c|c|c|c|c|}
\hline & $\begin{array}{c}\text { All } \\
(n=92)\end{array}$ & $\begin{array}{c}\text { Non-frail group } \\
(\text { KCL score }<8, n=29)\end{array}$ & $\begin{array}{c}\text { Frail group } \\
(\text { KCL } \text { score } \geq 8, n=63)\end{array}$ & $P$ \\
\hline Age (years) & $81.7 \pm 6.6$ & $79.1 \pm 7.6$ & $83.1 \pm 6.1$ & 0.019 \\
\hline Gender (male/female) & $54 / 38$ & $21 / 8$ & $33 / 30$ & 0.124 \\
\hline BMI $\left(\mathrm{kg} / \mathrm{m}^{2}\right)$ & $22.1 \pm 4.1$ & $24.1 \pm 3.6$ & $21 \pm 3.3$ & 0.001 \\
\hline Atrial fibrillation (\%) & 33 & 35 & 32 & 0.842 \\
\hline $\begin{array}{l}\text { KCL score } \\
\text { Robust/Pre-frail }(n)\end{array}$ & $10.7 \pm 5.7$ & $\begin{array}{l}4.2 \pm 2.2 \\
6 / 23\end{array}$ & $13.3 \pm 4.0$ & $<0.001$ \\
\hline \multicolumn{5}{|l|}{$\mathrm{J}-\mathrm{CHS}$} \\
\hline Robust/Pre-frail (n) & & $3 / 44$ & 45 & \\
\hline Cardiomyopathy (\%) & 12 & 8 & 14 & 0.377 \\
\hline Ischemic heart disease (\%) & 27 & 38 & 23 & 0.259 \\
\hline Hypertension (\%) & 9 & 4 & 11 & 0.213 \\
\hline Tachycardia-induced (\%) & 17 & 23 & 15 & 0.192 \\
\hline Valve $(\%)$ & 11 & 4 & 14 & 0.113 \\
\hline Pacemaker (\%) & 11 & 4 & 14 & 0.113 \\
\hline Other $(\%)$ & 13 & 19 & 9 & 0.127 \\
\hline \multicolumn{5}{|l|}{ Medication } \\
\hline Diuretic (\%) & 55 & 39 & 64 & 0.073 \\
\hline Tolvaptan (\%) & 22 & 22 & 23 & 0.876 \\
\hline ACE-I/ARB (\%) & 52 & 52 & 51 & 0.854 \\
\hline$\beta$ blocker (\%) & 31 & 22 & 32 & 0.412 \\
\hline Spironolactone $(\%)$ & 24 & 22 & 23 & 0.876 \\
\hline Anticoagulant (\%) & 38 & 39 & 38 & 0.951 \\
\hline \multicolumn{5}{|l|}{ Clinical data } \\
\hline $\operatorname{LVEF}(\%)$ & $57.8 \pm 14.3$ & $62.1 \pm 9.4$ & $55.7 \pm 15.9$ & 0.082 \\
\hline E/e' & $16.0 \pm 7.7$ & $15.1 \pm 7.2$ & $16.1 \pm 6.7$ & 0.534 \\
\hline $\mathrm{LAD}(\mathrm{mm})$ & $39.3 \pm 6.6$ & $40.7 \pm 8.3$ & $39.1 \pm 6.1$ & 0.402 \\
\hline $\mathrm{WBC}\left(/ \mathrm{mm}^{3}\right)$ & $5718 \pm 1923$ & $5732 \pm 1631$ & $5940 \pm 2232$ & 0.741 \\
\hline $\mathrm{Hb}(\mathrm{g} / \mathrm{dL})$ & $12.1 \pm 2.0$ & $13.3 \pm 1.9$ & $11.5 \pm 1.9$ & 0.001 \\
\hline $\mathrm{TP}(\mathrm{g} / \mathrm{dL})$ & $6.9 \pm 0.6$ & $7.2 \pm 0.5$ & $6.7 \pm 0.6$ & 0.547 \\
\hline Alb (g/dL) & $3.8 \pm 0.5$ & $4 \pm 0.3$ & $3.6 \pm 0.6$ & $<0.001$ \\
\hline T. chol (mg/dL) & $175 \pm 36$ & $186 \pm 32$ & $173 \pm 37$ & 0.164 \\
\hline $\mathrm{TG}(\mathrm{mg} / \mathrm{dL})$ & $111 \pm 54$ & $120 \pm 59$ & $107 \pm 68$ & 0.637 \\
\hline HbAlc (\%) & $6.1 \pm 0.7$ & $6.1 \pm 0.4$ & $6.2 \pm 0.8$ & 0.602 \\
\hline $\mathrm{BNP}(\mathrm{pg} / \mathrm{mL})$ & $173 \pm 178$ & $123 \pm 144$ & $222 \pm 195$ & 0.038 \\
\hline eGFR (mL/minute/1.73 $\left.\mathrm{m}^{2}\right)$ & $50 \pm 19$ & $56 \pm 14$ & $48 \pm 23$ & 0.101 \\
\hline \multicolumn{5}{|l|}{ CPX data } \\
\hline RER & $1.09 \pm 0.07$ & $1.09 \pm 0.05$ & $1.09 \pm 0.08$ & 0.599 \\
\hline Peak $\mathrm{VO}_{2}$ & $13.2 \pm 3.8$ & $15.1 \pm 4.5$ & $12.1 \pm 2.9$ & 0.006 \\
\hline Resting HR (bpm) & $72 \pm 12$ & $74 \pm 10$ & $71 \pm 13$ & 0.429 \\
\hline Peak HR (bpm) & $109 \pm 19$ & $117 \pm 21$ & $105 \pm 17$ & 0.021 \\
\hline Resting SBP (mmHg) & $136 \pm 22$ & $143 \pm 18.9$ & $132 \pm 25$ & 0.078 \\
\hline Peak SBP (mmHg) & $180 \pm 34$ & $192 \pm 38$ & $174 \pm 32$ & 0.044 \\
\hline
\end{tabular}

BMI indicates body mass index; KCL, Kihon checklist; J-CHS, the Japanese version of the Cardiovascular Health Study; HR, heart rate; ACE-I, angiotensin converting enzyme inhibitor; ARB, angiotensin receptor blocker; LVEF, left ventricular ejection fraction; E/e', ratio of early transmitral flow velocity to early diastolic mitral annular velocity; LAD, left atrium dimension; WBC, white blood cell; Hb, hemoglobin; TP, total protein; Alb, albumin; T. Chol, total cholesterol; TG, triglyceride; HbA1c, glycohemoglobin; BNP, brain natriuretic peptide; eGFR, estimated glomerular filtration rate; RER, respiratory exchange ratio; WR, work rate; CPX, cardiopulmonary exercise testing; and SBP, systolic blood pressure.

lapping pathophysiology between frailty and HF.

In the present study, the average age of the subjects was 81.7 years, $68.5 \%$ were classified as frail (KCL score $\geq 8$ ), and the average plasma BNP was $182 \mathrm{pg} / \mathrm{mL}$ once they had been stabilized after admission. We consider that these values are standard level of the elderly population who is admitted to hospital due to worsening HF in Japan nowadays. Frail patients had a significantly higher age and significantly lower body mass index than non-frail patients, which is consistent with the general frailty con- cept. ${ }^{1)}$ Echocardiogram parameters, such as left ventricular ejection fraction and left arterial dimension, did not differ between patients with and without frailty. In the case of left ventricular ejection fraction, this finding is not surprising given that approximately half of all patients with $\mathrm{HF}$ have preserved ejection fraction. ${ }^{13)}$ Among the biomarkers examined, BNP, suggested to be a strong, independent predictor of cardiac events, ${ }^{14)}$ was significantly greater in frail patients than in non-frail patients, suggesting that frailty may worsen $\mathrm{HF}$ independent of systolic 


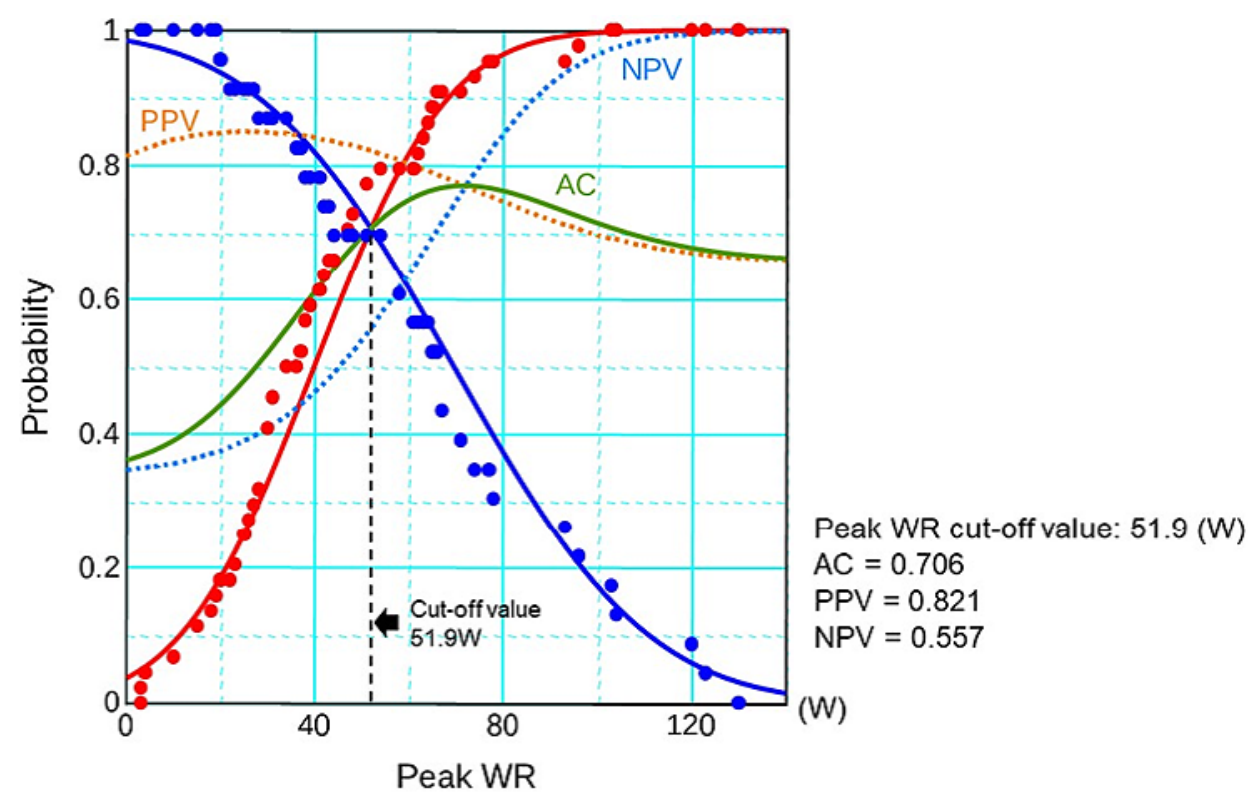

Figure 2. Diagnostic performance plot showing peak WR's ability to detect frailty defined as a KCL score $\geq 8$. AC indicates accuracy; KCL, Kihon Checklist; NPV, negative predictive value; PPV, positive predictive value; and WR, work rate. A diagnostic performance plot analysis was conducted to assess peak WR's utility to distinguish between the presence (KCL score $\geq 8$ ) or absence of frailty. Cumulative frequency distributions were generated to determine the optimal cutoff point for detecting frailty. Sensitivity, specificity, accuracy, and positive predictive value were calculated using standard formulas.

Table II. Correlations between CPX Parameter and KCL Score

\begin{tabular}{lcr}
\hline \multicolumn{1}{c}{ CPX parameter } & \multicolumn{1}{c}{$r$} & \multicolumn{1}{c}{$P$} \\
\hline RER & -0.07 & 0.571 \\
WR at AT $(\mathrm{W})$ & -0.557 & $<0.001$ \\
Peak WR $(\mathrm{W})$ & -0.632 & $<0.001$ \\
$\mathrm{VO}_{2}$ at $\mathrm{AT}(\mathrm{mL} /$ minute $/ \mathrm{kg})$ & -0.291 & 0.019 \\
Peak $_{2}(\mathrm{~mL} / \mathrm{minute} / \mathrm{kg})$ & -0.527 & $<0.001$ \\
$\mathrm{VE} / \mathrm{VCO}_{2}$ slope & 0.435 & $<0.001$ \\
$\Delta \mathrm{VO} / \mathrm{WWR}$ & -0.621 & $<0.001$ \\
Resting SBP $(\mathrm{mmHg})$ & -0.165 & 0.182 \\
Peak SBP $(\mathrm{mmHg})$ & -0.37 & 0.002 \\
\hline
\end{tabular}

AT indicates anaerobic threshold; and VE, minutes of ventilation. Other abbreviations are as in Table I.

function.

In the present study, all the CPX parameters during exercise were significantly correlated with KCL score (Tables II, III). In addition, the CPX parameters had generally stronger correlations with KCL score compared with the resting biomarkers and echocardiogram parameters. Of the CPX parameters examined, peak WR was superior to peak $\mathrm{VO}_{2}$ as a diagnostic marker of increased KCL score. Ultimately, we found that peak WR and peak SBP were significant independent predictors of frailty (Table III) and that a peak WR less than around $50 \mathrm{~W}$ could indicate the presence of frailty in elderly patients with stable HF.

Peak WR is one of the most powerful prognostic predictors used in HF management. ${ }^{15)}$ Peak WR is a combined measure of muscular strength and endurance. Subjective symptoms in daily activity, particularly dyspnea on
Table III. Results of the Multivariate Analysis with KCL Score

\begin{tabular}{lcrr}
\hline & $\beta$ & $95 \% \mathrm{CI}$ & \multicolumn{1}{c}{$P$} \\
\hline Peak WR & -0.108 & -0.144 to -0.072 & $<0.001$ \\
Peak SBP & -0.045 & -0.074 to -0.016 & 0.003 \\
\hline After adjusting WR at $\mathrm{AT}, \mathrm{VO}_{2}$ at $\mathrm{AT}$, peak $\mathrm{VO}_{2}$, \\
VE/VCO $\mathrm{VH}_{2}$ slope, and $\Delta \mathrm{VO}_{2} / \Delta \mathrm{WR}$. Abbreviations are \\
as in Tables I and II.
\end{tabular}

exertion, are due to a lack of muscular endurance in patients with chronic HF. ${ }^{16)}$ Muscular strength and endurance, combined with quadriceps muscle area, are also major predictors of peak WR in patients with chronic HF. ${ }^{17)}$ Frailty likely affects muscle activity rather than cardiac output; suggesting that peak WR is more useful than other $\mathrm{CPX}$ variables, including peak $\mathrm{VO}_{2}$, for assessing frailty status in elderly patients with HF.

We also found that peak SBP was an independent predictor of frailty. It has been reported that reduced exercise duration and peak SBP are independent predictors of all-cause mortality in ambulatory patients with chronic $\mathrm{HF}^{18)}$ Similarly, exercise cardiac power (a non-invasive surrogate for cardiac power output), which is the product of peak $\mathrm{VO}_{2}$ and peak $\mathrm{SBP},{ }^{19)}$ has been reported to be predictive of prognosis in HF. ${ }^{19)}$ These findings imply the importance of assessing cardiac pumping capability during exercise in patients with $\mathrm{HF}^{20)}$ In contrast to peak SBP, we found no correlation between resting SBP and KCL score; therefore, the relationships among resting and peak SBP and frailty status remain unclear, and further prospective studies are warranted. 
The recognition of frailty within the medical community has created the need for diagnostic tests to determine when a patient's physical ability has deteriorated. CPX is a non-invasive diagnostic tool that can be used to detect changes in exercise capacity over time. We found that using CPX to assess peak WR, rather than assessing resting echocardiography parameters or biomarkers, could be useful for detecting frailty in elderly patients with stable HF. To our knowledge, this is the first study to examine the use of CPX variables to assess frailty status. The primary goals of HF therapy are to improve quality of life, reduce exacerbation frequency, and extend survival. Currently, there is no specific therapy for HF patients with frailty. Although we considered the frail patients in the present study to be optimally medicated, based on current HF guidelines,${ }^{21)}$ they were often receiving polypharmacy, which is problematic because age-related changes in absorption and metabolism alter most drugs' pharmacokinetics. ${ }^{22}$ Using CPX to detect frailty could not only lead to early therapeutic intervention but also provide safe, effective exercise training based on an exercise prescription.

This was a single-center study with a small sample size. Moreover, we did not assess repeated measures over time or investigate the incidence of cardiac events in enrolled patients. We also did not assess changes in the trajectory of exercise capacity or frailty due to medical intervention or cardiac rehabilitation. Cardiac rehabilitation is a comprehensive, secondary prevention program designed to optimize physical activity and health in the context of known disease; as such, it provides important opportunities to address the idiosyncratic needs of elderly patients with $\mathrm{HF}^{23,24)}$ Of note, not all elderly HF patients can undertake CPX because a certain amount of physical ability is required.

\section{Conclusion}

The present study showed that peak WR and peak SBP during exercise were strongly associated with frailty status in elderly patients with stable HF. Peak $\mathrm{VO}_{2}, \mathrm{VE} /$ $\mathrm{VCO}_{2}$ slope, and $\Delta \mathrm{VO}_{2} / \Delta \mathrm{WR}$ were also associated with frailty status in this population. Thus, CPX variables could be useful for diagnosing frailty in elderly patients with stable $\mathrm{HF}$

\section{Acknowledgments}

The authors are indebted to the staff members of the National Center for Geriatrics and Gerontology, Obu, Japan, particularly Kimiko Hori (physiological technician) and Naoki Ito, Kenji Sato, and Kakeru Hashimoto (physical therapists), and Ikue Ueda (occupational therapist).

\section{Disclosure}

Conflicts of interest: There are no conflicts of interest to declare.

\section{References}

1. Fried LP, Tangen CM, Walston J, et al. Frailty in older adults: evidence for a phenotype. J Gerontol A Biol Sci Med Sci 2001; 56: M146-56.

2. Afilalo J. Frailty in patients with cardiovascular disease: Why, When, and How to Measure. Curr Cardiovasc Risk Rep 2011; 5: 467-72.

3. Satake S, Shimada H, Yamada M, et al. Prevalence of frailty among community-dwellers and outpatients in Japan as defined by the Japanese version of the Cardiovascular Health Study criteria. Geriatr Gerontol Int 2017; 17: 2629-34.

4. Balady GJ, Arena R, Sietsema K, et al. Clinician's Guide to cardiopulmonary exercise testing in adults: a scientific statement from the American Heart Association. Circulation 2010; 122: 191-225.

5. Guazzi M, Myers J, Peberdy MA, et al. Echocardiography with Tissue Doppler Imaging and cardiopulmonary exercise testing in patients with heart failure: a correlative and prognostic analysis. Int J Cardiol 2010; 143: 323-9.

6. Yamada T, Hirashiki A, Okumura T, et al. Prognostic impact of combined late gadolinium enhancement on cardiovascular magnetic resonance and peak oxygen consumption in ambulatory patients with nonischemic dilated cardiomyopathy. J Card Fail 2014; 20: 825-32.

7. Takagawa Y, Yagi S, Ise T, et al. Improved exercise capacity after cardiac rehabilitation is associated with reduced visceral fat in patients with chronic heart failure. Int Heart J 2017; 58: 74651 .

8. Arai H, Satake S. English translation of the Kihon Checklist. Geriatr Gerontol Int 2015; 15: 518-9.

9. Satake S, Senda K, Hong YJ, et al. Validity of the Kihon Checklist for assessing frailty status. Geriatr Gerontol Int 2016; 16: 709-15.

10. ATS/ACCP Statement on cardiopulmonary exercise testing. Am J Respir Crit Care Med 2003; 167: 211-77.

11. Hunt SA. ACC/AHA 2005 guideline update for the diagnosis and management of chronic heart failure in the adult: a report of the American College of Cardiology/American Heart Association Task Force on Practice Guidelines (Writing Committee to Update the 2001 Guidelines for the Evaluation and Management of Heart Failure). J Am Coll Cardiol 2005; 46: e1-82.

12. Sewo Sampaio PY, Sampaio RA, Yamada M, Arai H. Systematic review of the Kihon Checklist: Is it a reliable assessment of frailty? Geriatr Gerontol Int 2016; 16: 893-902.

13. Hogg K, Swedberg K, McMurray J. Heart failure with preserved left ventricular systolic function; epidemiology, clinical characteristics, and prognosis. J Am Coll Cardiol 2004; 43: 317-27.

14. Berger R, Huelsman M, Strecker K, et al. B-type natriuretic peptide predicts sudden death in patients with chronic heart failure. Circulation 2002; 105: 2392-7.

15. Dickstein K, Cohen-Solal A, Filippatos G, et al. ESC Guidelines for the diagnosis and treatment of acute and chronic heart failure 2008: the Task Force for the Diagnosis and Treatment of Acute and Chronic Heart Failure 2008 of the European Society of Cardiology. Developed in collaboration with the Heart Failure Association of the ESC (HFA) and endorsed by the European Society of Intensive Care Medicine (ESICM). Eur Heart J 2008; 29: 2388-442.

16. Suzuki K, Omiya K, Yamada S, et al. Relations between strength and endurance of leg skeletal muscle and cardiopulmonary exercise testing parameters in patients with chronic heart failure. J Cardiol 2004; 43: 59-68.

17. Senden PJ, Sabelis LW, Zonderland ML, et al. Determinants of maximal exercise performance in chronic heart failure. Eur $\mathrm{J}$ Cardiovasc Prev Rehabil 2004; 11: 41-7.

18. Williams SG, Jackson M, Ng LL, Barker D, Patwala A, Tan LB. Exercise duration and peak systolic blood pressure are predictive of mortality in ambulatory patients with mild-moderate chronic heart failure. Cardiology 2005; 104: 221-6. 
19. Cohen-Solal A, Tabet JY, Logeart D, Bourgoin P, Tokmakova M, Dahan M. A non-invasively determined surrogate of cardiac power ('circulatory power') at peak exercise is a powerful prognostic factor in chronic heart failure. Eur Heart J 2002; 23: 80614.

20. Tan LB, Bain RJ, Littler WA. Assessing cardiac pumping capability by exercise testing and inotropic stimulation. Br Heart J 1989; 62: 20-5.

21. Ponikowski P, Voors AA, Anker SD, et al. 2016 ESC Guidelines for the diagnosis and treatment of acute and chronic heart failure: The Task Force for the diagnosis and treatment of acute and chronic heart failure of the European Society of Cardiology
(ESC) Developed with the special contribution of the Heart Failure Association (HFA) of the ESC. Eur Heart J 2016; 37: 2129-200.

22. Veronese N, Stubbs B, Noale M, et al. Polypharmacy is associated with higher frailty risk in older people: An 8-year longitudinal cohort study. J Am Med Dir Assoc 2017; 18: 624-8.

23. Menezes AR, Lavie CJ, Forman DE, Arena R, Milani RV, Franklin BA. Cardiac rehabilitation in the elderly. Prog Cardiovasc Dis 2014; 57: 152-9.

24. Forman DE, Rich MW, Alexander KP, et al. Cardiac care for older adults. Time for a new paradigm. J Am Coll Cardiol 2011; 57: 1801-10. 